Let $p$ be a positive prime number, and $T_{p}$ be the semigroup of all positive rational numbers of the form $j / p^{i}$ with respect to the usual addition. Let $p_{1}, p_{2}, \cdots, p_{n}$ be distinct positive prime numbers. Then $T_{p_{1}} \times \cdots \times T_{p_{n}}$ is a completely exclusive direct product.

\title{
REFERENCES
}

1. A. H. Clifford, Naturally totally ordered commutative semigroups, Amer. J. Math. 76 (1954), 631-646.

2. - Totally ordered commutative semigroups, Bull. Amer. Math. Soc. 64 (1958), 305-316.

3. O. Hölder, Die Axiome der Quantität und die Lehre vom Mass, Ber. Verh. Sächs. Ges. Wiss. Leipzig Math.-Phys. Kl. 53 (1901), 1-64.

4. T. Tamura, Commutative nonpotent archimedean semigroup with cancellation law. I, J. Gakugei Tokushima Univ. 8 (1957), 5-11.

University of California, Davis

\section{THE INDEX OF ELLIPTIC OPERATORS ON COMPACT MANIFOLDS}

\author{
BY M. F. ATIYAH AND I. M. SINGER ${ }^{1}$
}

Communicated by Raoul Bott, February 1, 1963

Introduction. In his paper [16] Gel'fand posed the general problem of investigating the relationship between topological and analytical invariants of elliptic differential operators. In particular he suggested that it should be possible to express the index of an elliptic operator (see $\$ 1$ for the definition) in topological terms. This problem has been taken up by Agranovic $[2 ; 3]$, Dynin $[3 ; 14 ; 15]$, Seeley $[20 ; 21]$ and Vol'pert [22] who have solved it in special cases. The purpose of this paper is to give a general formula for the index of an elliptic operator on any compact oriented differentiable manifold (Theorem 1). As a special case of this formula we get the Hirzebruch-RiemannRoch theorem for any compact complex manifold (Theorem 3). This was previously known only for projective algebraic manifolds. Some other special cases, of interest in differential topology, are discussed in $\$ 3$.

We are greatly indebted to A. P. Calderon, L. Nirenberg, and R. T. Seeley for their generous help.

1. Elliptic operators. Let $X$ be a compact oriented smooth mani-

${ }^{1}$ Part of this work was done with the first author supported by the National Science Foundation and the second author holding a Sloan Fellowship. 
fold, $E, F$ two smooth complex vector bundles over $X$. We shall consider linear differential operators

$$
D: \Gamma(E) \rightarrow \Gamma(F),
$$

i.e., linear operators defined on the spaces $\Gamma$ of smooth sections and expressible locally by a matrix of partial derivatives. The extra generality involved in considering vector bundles presents no serious difficulties and it is quite essential in a systematic treatment on manifolds, since all the geometrically interesting operators operate on vector bundles (cf. \$3).

Let $T^{*}(X)$ denote the cotangent vector bundle of $X, S(X)$ the unit sphere bundle in $T^{*}(X)$ (relative to some Riemannian metric), $\pi: S(X) \rightarrow X$ the projection. Then associated with $D$ there is a vector bundle homomorphism

$$
\sigma(D): \pi^{*} E \rightarrow \pi^{*} F
$$

which is called the symbol of $D$. In terms of local coordinates $\sigma(D)$ is obtained from $D$ by replacing $\partial / \partial x_{j}$ by $i \xi_{j}$ in the highest order terms of $D\left(\xi_{j}\right.$ is the $j$ th coordinate in the cotangent bundle). $D$ is elliptic if $\sigma(D)$ is an isomorphism. ${ }^{2}$

One of the basic properties of elliptic operators is that $\operatorname{Ker} D$ (i.e., the null space) and Coker $D=\Gamma(F) / D \Gamma(E)$ are both finite-dimensional. The index $\gamma(D)$ is defined by

$$
\gamma(D)=\operatorname{dim} \operatorname{Ker} D-\operatorname{dim} \text { Coker } D .
$$

If $D^{*}: \Gamma(F) \rightarrow \Gamma(E)$ denotes the formal adjoint of $D$ (relative to metrics in $E, F, X)$ then $D^{*}$ is also elliptic and

$$
\text { Coker } D \cong \operatorname{Ker} D^{*} \text {, }
$$

so that $\gamma(D)=\operatorname{dim} \operatorname{Ker} D-\operatorname{dim} \operatorname{Ker} D^{*}$.

The problem is to express $\gamma(D)$ in terms of $\sigma(D)$. More precisely what we shall do is to associate to $\sigma(D)$ a cohomological invariant, denoted by $\operatorname{ch}(D)$, and then to give an explicit formula for $\gamma(D)$ in terms of $\operatorname{ch}(D)$ and of the characteristic (Pontrjagin) classes of $X$.

2. The index formula. If $E, F$ are trivial bundles of dimension $m$ then $\sigma(D)$ is essentially a map $S(X) \rightarrow G L(m, C)$. This makes it clear that the homotopy of the linear group, and in particular the Bott periodicity theorems [8], will be of crucial importance. The appropriate machinery for questions of this kind is provided by the Grothendieck groups $K(X)$ introduced in [5] (cf. also [4]). Roughly speaking $K(X)$ is the abelian group generated by the complex vector bundles

2 This implies of course that $E, F$ have the same dimension. 
on $X$. The tensor product of bundles makes $K(X)$ into a commutative ring. An important cohomological invariant is provided by the Chern character which is a ring homomorphism

$$
\text { ch: } K(X) \rightarrow H^{*}(X ; \mathbf{Q})
$$

of $K(X)$ into the rational cohomology ring.

Two vector bundles $E, F$ on a space $Y$ with an isomorphism $\sigma$ on a subspace ${ }^{3} Y_{0}$ define a difference element $[6, \S 3]$

$$
d(E, F, \sigma) \in K\left(Y / Y_{0}\right)
$$

where $Y / Y_{0}$ is $Y$ with $Y_{0}$ pinched to a point. Hence, if we denote by $B(X)$ the unit ball bundle of $T^{*}(X)$, an elliptic operator $D$ defines an element

$$
d\left(p^{*} E, p^{*} F, \sigma(D)\right) \in K(B(X) / S(X))
$$

(where $p: B(X) \rightarrow X$ is the projection) and hence an element

$$
\operatorname{ch} d(E, F, \sigma(D)) \in H^{*}(B(X) / S(X) ; Q) \text {. }
$$

Using the Thom isomorphism

$$
\phi_{*}: H^{k}(X ; Q) \cong H^{n+k}(B(X) / S(X) ; Q) \quad(n=\operatorname{dim} X)
$$

we obtain finally the element

$$
\phi_{*^{-1}} \operatorname{ch} d(E, F, \sigma(D)) \in H^{*}(X ; Q)
$$

which we shall simply denote by $\operatorname{ch} \sigma(D)$ or just $\operatorname{ch}(D)$.

We recall next the definition of the Todd class. For any $n$-dimensional complex vector bundle $\xi$ we define $\Im(\xi)$ by putting

$$
J(\xi)=\prod_{i=1}^{n} \frac{x_{i}}{1-e^{-x_{i}}} \quad \text { where } \operatorname{ch} \xi=\sum_{i=1}^{n} e^{x_{i}}
$$

and the $x_{i}$ are of degree 2 . The elementary symmetric functions of the $x_{i}$ are the Chern classes $c_{i}(\xi)$, so that $J(\xi)$ is given by a power series in the $c_{i}(\xi)$. For a real vector bundle $\eta$ we define

$$
J(\eta)=J\left(\eta \otimes_{R} C\right)
$$

so that $J(\eta)$ will be given by a power series in the Chern classes of $\eta \otimes_{R} C$, i.e., in the Pontrjagin classes of $\eta$. For a differentiable manifold $^{4} X$ we define

${ }^{3}$ We assume $Y_{0}$ a "reasonable" subspace, e.g., a subcomplex.

${ }^{4}$ If $Y$ is a complex manifold with $X$ as underlying differentiable manifold, one must not confuse $T(Y)$ with $T(X) \otimes_{R} C$. 


$$
\Im(X)=\Im(T(X))
$$

where $T(X)$ is the tangent bundle of $X$. Thus, if the Pontrjagin classes of $X$ are as usual regarded as the elementary symmetric functions in $y_{j}^{2}$, the Todd class is given by:

$$
J(X)=\prod_{j} \frac{y_{j}}{1-e^{-y_{j}}} \cdot \frac{-y_{j}}{1-e^{y_{j}}} .
$$

For any $\alpha \in H^{*}(X ; Q)$ we denote by $\alpha[X]$ the value of the topdimensional component of $\alpha$ on the fundamental homology class of $X$.

With these definitions we can now state our main result:

THEOREM 1. For any elliptic differential operator $D$ on a compact oriented differentiable manifold $X$ the index $\gamma(D)$ is given by the formula

$$
\gamma(D)=\{\operatorname{ch}(D) \cdot \Im(X)\}[X] \text {. }
$$

REMARKS. 1. This formula applies also to elliptic singular integral operators (cf. \$4).

2. In some special cases one can deduce from Theorem 1 that $\gamma(D)=0$ (cf. (3.5)).

3. The right-hand side of this equation gives, a priori, only a rational number. It is a consequence of the theorem that this number must be an integer (cf. \$3).

3. Special cases. The most interesting differential operators are those associated with some geometrical structure on $X$ in the following way. Let $G$ be a Lie group, $V$ a fixed real oriented $G$-module, $V^{*}$ its dual. Then a $G$-structure on $X$ will mean a principal $G$-bundle $P$ on $X$ together with an isomorphism (of oriented bundles)

$$
P \times_{a} V \cong T(X)
$$

where $P \times_{G} V$ is the vector bundle associated to $P$ by the $G$-module $V$. A differential operator $D: \Gamma(E) \rightarrow \Gamma(F)$ of order $k$ is said to be associated to the G-structure of $X$ if

(i) there are complex $G$-modules $M, N$ and isomorphisms

$$
P \times_{G} M \cong E, \quad P \times_{G} N \cong F,
$$

(ii) there is a $G$-map

$$
V^{*} \rightarrow \operatorname{Hom}(M, N)
$$

which is polynomial of degree $k$ and, via the isomorphisms (A) and (B), induces the symbol $\sigma(D)$.

For such operators we have the following theorem which gives a universal formula for $\operatorname{ch} D$. 
TheOREM 2. Let $X$ have a G-structure, $\operatorname{dim} V=2 l$, let the image of $G$ in Aut $V$ have rank $l$, and suppose $D: \Gamma(E) \rightarrow \Gamma(F)$ is an elliptic operator associated to the $G$-structure. Then $\operatorname{ch} D$ is the characteristic class of the G-structure corresponding to the universal class

$$
(\operatorname{ch} M-\operatorname{ch} N) \prod_{i=1}^{l} \omega_{i}^{-1} \in H^{* *}\left(B_{G} ; \boldsymbol{Q}\right)
$$

where the $\omega_{i}$ are the negative ${ }^{5}$ weights of the real G-module $V$, ch $M$ and $\operatorname{ch} N$ denote the characters of these complex G-modules and we use the Borel-Hirzebruch method [7] of describing the cohomology of the classifying space $B_{G}$.

REMARK. This shows that $\operatorname{ch} D$ depends, in this case, only on $E, F$ and not on the particular $D$.

In (3.1)-(3.3) below we give examples of such operators. In all cases $k=1$, i.e., $D$ is of first order, and the ellipticity is easily verified.

(3.1) Riemannian Structure. $G=S O(2 l), V=R^{2 l}$.

Let $\Lambda=\sum_{p=0}^{2 l} \Lambda^{p}$ denote the complexification of the exterior algebra of $V^{*}$. Let $*$ be the usual dualizing transformation of $\Lambda$; thus $*: \Lambda^{p} \rightarrow \Lambda^{2 l-p}$ is an isomorphism and $(*)^{2}=(-1)^{p}$ when applied to $\Lambda^{p}$. Let $\alpha: \Lambda^{p} \rightarrow \Lambda^{2 l-p}$ be defined by $\alpha=i^{p(p+1)-l_{*}}$ so that $\alpha^{2}=1$. Now take $M(N)$ to be the $+1(-1)$ eigenspace of the involution $\rho$ of $\Lambda$ in the two cases
(i) $\rho=(*)^{2}$,
(ii) $\rho=\alpha$.

In each case we take $D=d+\delta$ where, as usual, $d$ denotes the exterior derivative of forms and $\delta$ is its formal adjoint. It is easily verified that for any form $\omega$

$$
\rho \omega=\omega \Rightarrow \rho(D \omega)=-D \omega,
$$

so that $D: \Gamma(E) \rightarrow \Gamma(F)$ is defined. Theorems 1, 2 applied in these two cases give

(i) $\sum_{p=0}^{2 l}(-1)^{p} h^{p}=\chi[X]$, where $h^{p}=\operatorname{dim} H^{p}, H^{p}$ is the space of harmonic $p$-forms and $\chi$ is the Euler class of $X$;

(ii) $h_{+}^{l}-h_{-}^{l}=0$ if $l$ is odd $=L(X)$, the Hirzebruch $L$-genus [17], if $l$ is even, where $h_{+}^{l}=\operatorname{dim} H_{+}^{l}, h_{-}^{l}=\operatorname{dim} H_{-}^{l}$ and $H^{l}=H_{+}^{l} \oplus H_{-}^{l}$ is the decomposition into the eigenspaces of $\alpha$.

Case (i) is an elementary consequence of the Hodge theory and the

5 The "negative" weights depend on some choices, but their product depends only on the orientation of $V$ which is fixed. 
Gauss-Bonnet formula. Case (ii) is the Hirzebruch index theorem [17, $\S 8$ ], since $h_{+}^{l}-h_{-}^{l}$ is, for $l$ even, easily seen to be the index (or signature) of the quadratic form in $H^{l}$ given by the cup-product.

REMARK. Operating on the space of all forms $d+\delta$ is formally selfadjoint and so has index zero. We get an interesting index therefore only by decomposing the space of forms and restricting the domain of $d+\delta$ as above. The same remark applies to the next two examples.

(3.2) Hermitian structure. $G=U(l) \times U(m), V=C^{l}$.

We start now with a complex manifold $X$ of complex dimension $l$, and a holomorphic vector bundle $W$ of dimension $m$, both with Hermitian metrics. Let $\Lambda=\sum_{p=0}^{l} \Lambda^{p}$ denote the exterior algebra of $V$ and define the $G$-modules

$$
M=\left(\sum_{k} \Lambda^{2 k}\right) \otimes C^{m}, \quad N=\left(\sum_{k} \Lambda^{2 k+1}\right) \otimes C^{m} .
$$

Since $V \cong \bar{V}^{*}$ as $G$-modules we can interpret $\Gamma(E), \Gamma(F)$ as spaces of forms of type $(0, p)$ with coefficients in $W$. We take $D=\bar{\partial}+\delta$ where, as usual, $\bar{\partial}$ is the $(0,1)$ component of the exterior derivative and $\boldsymbol{b}$ is its formal adjoint. The index of $D$ is now

$$
\gamma(D)=\sum_{p=0}^{l}(-1)^{p} h^{0, p}(W)
$$

where $h^{0, p}(W)=\operatorname{dim} H^{0, p}(W)$ and $H^{0, p}(W)$ denotes the space of harmonic forms of type $(0, p)$ with coefficients in $W$ (relative to the Laplacian $\bar{\partial} \delta+\delta \bar{\partial})$. But by the Dolbeault isomorphism $[17, \S 15]$ we have

$$
H^{0, p}(W) \cong H^{p}(X, W)
$$

where $H^{p}(X, W)$ denotes the $p$-dimensional cohomology group of $X$ with coefficients in the sheaf of germs of holomorphic sections of $W$. Applying Theorems 1, 2 we get the Hirzebruch-Riemann-Roch theorem:

Theorem 3. For any compact complex manifold $X$ and any holomorphic vector bundle $W$ we have

$$
\sum(-1)^{p} \operatorname{dim} H^{p}(X, W)=\{\operatorname{ch}(W) J(X)\}[X],
$$

where $\Im(X)$ denotes the Todd class of the (complex) tangent bundle of $X$.

This theorem was proved for projective algebraic manifolds by Hirzebruch [17] and, in a weaker form, by Kodaira [18] for Kahler surfaces. 
(3.3) Spinor structure. $G=\operatorname{Spin}(2 l), V=R^{2 l}$.

We take $M=S^{+}, N=S^{-}$to be the two half-spin representations. The Dirac operator $D$ is a first order differential operator on spinor fields given, in terms of an orthonormal base $\left\{e_{i}\right\}$ of the tangent space, by

$$
D s=\sum_{i} e_{i} \partial_{i}(s)
$$

where $\partial_{i}(s)$ is the covariant derivative in the $i$ th direction and $e_{i}$ operates on spinors by Clifford multiplication (cf. $[10, \$ 10]$ ). $D$ interchanges positive and negative spinors. Let $H(S)$ denote the space of all harmonic spinors (relative to the Laplacian $D^{2}$ ), $H(S)$ $=H\left(S^{+}\right) \oplus H\left(S^{-}\right)$the decomposition into positive and negative spinors, and put

$$
h^{+}(S)=\operatorname{dim} H\left(S^{+}\right), \quad h^{-}(S)=\operatorname{dim} H\left(S^{-}\right) .
$$

Then, applying Theorems 1,2 we get

THEOREM 4. Let $X$ be a manifold of even dimension with a spinorstructure. Then

$$
h^{+}(S)-h^{-}(D)=A(X)
$$

where $\hat{A}(X)$ is the Hirzebruch $\hat{A}$-genus [7].

REMARKs. (1) The results of (3.1) and (3.3) can, like (3.2), be generalized to forms or spinors with coefficients in a vector bundle.

(2) These results give an interesting analytical interpretation to the various "integrality theorems" for the $L$-genus, $\hat{A}$-genus and Todd genus (cf. [7]). All these integers now appear as the indices of elliptic operators. For example, it was known that the $\hat{A}$-genus was an integer for Spin-manifolds, but not for general manifolds. Theorem 4 provides an explanation of this.

(3.4) Homogeneous spaces. Consider a homogeneous space $X=H / G$ of a compact Lie group $H$, let $M, N$ be $G$-modules and $D$ any elliptic operator between the associated vector bundles which is invariant under the action of $H$. Then, by direct methods, using induced representations, Bott [9] has shown that

(i) if $\operatorname{rank} G<\operatorname{rank} H, \gamma(D)=0$,

(ii) if $\operatorname{rank} G=\operatorname{rank} H$ (so that $\operatorname{dim} H / G$ is even) the formula for $\gamma(D)$ given by Theorems 1,2 comes out of the Hermann Weyl character formula.

(3.5) CASES OF ZERO INDEX. In some cases one can prove that ch $D=0$ and so, from Theorem $1, \gamma(D)=0$. For example one can prove 
Proposition 1. Let $\operatorname{dim} X$ be odd and let $D$ be an elliptic operator ${ }^{6}$ such that, for $\xi \in S(X)$,

$$
\sigma(D)(-\xi) \cdot\{\sigma(S)(\xi)\}^{-1}
$$

depends only on $\pi(\xi) \in X$. Then $\operatorname{ch} D=0$.

An elliptic differential operator always fulfills the condition above (since $\sigma(D)(\xi)$ is then a homogeneous polynomial in $\xi$ ). Hence the index is zero in this case.

Proposition 2. Let $\operatorname{dim} X=n, D=\Gamma(E) \rightarrow \Gamma(F)$ an elliptic operator' where $E$ and $F$ are trivial bundles of dimension $m$. Then

(i) $(\operatorname{ch~} D)_{q}=0$ for $q>2 m-n$,

(ii) $(\operatorname{ch} D)_{0}=0$ if the Euler number of $X$ is nonzero.

From this, using Theorem 1, and the fact that Pontrjagin classes only occur in dimensions divisible by 4 we deduce that $\gamma(D)=0$ if

(a) $m<n / 2$ or

(b) $m=n / 2$ and $X$ has nonzero Euler number or $n \neq \equiv 0 \bmod 4$ or

(c) $m<n$ and $X$ has zero Pontrjagin classes in positive dimensions (e.g., $X$ a hypersurface in $R^{n+1}$ ).

The preceding cases of zero index include and generalize all the cases given in $[2 ; 3 ; 14 ; 15 ; 20 ; 22]$.

4. The group of elliptic symbols. If $E, F$ are two vector bundles on $X$ we have the set $\operatorname{Diff}(E, F)$ of all differential operators $D: \Gamma(E)$ $\rightarrow \Gamma(F)$. We can also consider the set $\operatorname{Int}(E, F)$ of all singular integral operators (cf. Seeley [19] and Dynin [14]), and we have a commutative diagram ${ }^{7}$

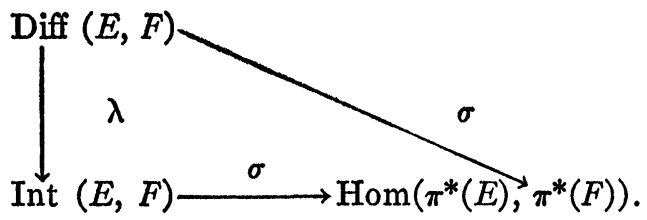

The bottom $\sigma$ (which is surjective) is a symbol given by a Fourier transform and the vertical map $\lambda$ is that of Calderon-Zygmund [11]. Ellipticity and the index $\gamma$ are defined for integral operators as for differential operators and one has

- Differential or integral (see $\$ 4$ ).

' Hom denotes here the set of all continuous vector bundle homomorphisms. 


$$
\gamma(D)=\gamma(\lambda(D)) \text {. }
$$

Moreover the index depends only on the symbol, so that we can regard $\gamma$ as an integer-valued function on Iso $\left(\pi^{*} E, \pi^{*} F\right)$ (the set of all continuous vector bundle isomorphisms). Moreover, one can show that

(i) $\gamma\left(\sigma \oplus \sigma^{\prime}\right)=\gamma(\sigma)+\gamma\left(\sigma^{\prime}\right)$,

(ii) $\gamma\left(\sigma \sigma^{\prime}\right)=\gamma(\sigma)+\gamma\left(\sigma^{\prime}\right)$,

(iii) $\gamma(\sigma)=0$ if $\sigma$ extends to an isomorphism $p^{*} E \rightarrow p^{*} F$ on $B(X)$.

Let us now define an equivalence relation on the set of all elliptic symbols by: $\sigma \sim \sigma^{\prime}$ if there exist $\alpha_{i}(i=1, \cdots, 4)$ which extend to $B(X)$ such that

$$
\sigma^{\prime} \oplus \alpha_{1}=\alpha_{2}\left(\sigma \oplus \alpha_{3}\right) \alpha_{4},
$$

and denote the set of equivalence classes by $\operatorname{Ell}(X)$. It is an abelian semi-group under $\oplus$ and (i)-(iii) show that $\gamma$ induces a homomorphism

$$
\gamma: \operatorname{Ell}(X) \rightarrow \boldsymbol{Z} .
$$

The function $\mu$ defined by

$$
\mu(\sigma)=\{\operatorname{ch}(\sigma) J(X)\}[X 1
$$

defines another homomorphism

$$
\mu: \operatorname{Ell}(X) \rightarrow \boldsymbol{Q}
$$

and Theorem 1 asserts that $\mu=\gamma$. The first main step in the proof is the determination of $\operatorname{Ell}(X)$ :

Proposition 3. $\operatorname{Ell}(X)$ is an abelian group and the tensor product makes it into a $K(X)$-module. If $\operatorname{dim} X$ is even, $D_{0}$ denotes the operator of (3.1) (ii), and $\sigma_{0}=\sigma\left(D_{0}\right)$ then $\operatorname{Ell}(X) / K(X) \sigma_{0}$ is a finite group.

This is proved by first showing that ${ }^{8} \operatorname{Ell}(X) \cong \widetilde{K}(B(X) / S(X))$, then applying the Chern character and observing that $\operatorname{ch}\left(\sigma_{0}\right)$ is an invertible element of $H^{*}(X ; Q)$.

REMARK. If $X$ admits either a complex or spinor structure then the symbols of the operators of (3.2) (with $W=1$ ) or (3.3) actually generate $\operatorname{Ell}(X)$ freely as a $K(X)$-module.

Proposition 3 reduces us to checking that $\gamma\left(W \sigma_{0}\right)=\mu\left(W \sigma_{0}\right)$ for all vector bundles $W$ on $X$. Here $\gamma\left(W \sigma_{0}\right)$ may be computed as the index of $D_{0}$ "with coefficients in $W$." Thus, for fixed $X$, we have replaced the general elliptic operator by one of this standard type. Having

${ }^{8} \tilde{K}$ denotes the "reduced" group of $K$ (cf. [5]). 
thus normalized our operators we propose next to vary $X$. To exhibit the dependence on $X$ we shall now write

$$
\mu\left(W \sigma_{0}\right)=\mu(X, W), \quad \gamma\left(W \sigma_{0}\right)=\gamma(X, W) .
$$

5. Cobordism. If $W=1$ the equality $\mu(X, 1)=\gamma(X, 1)$ is just the Hirzebruch index theorem (3.1) (ii). We shall therefore imitate Hirzebruch's proof $[17, \S 8]$ in our more general case. First we introduce the equivalence relation of cobordism on pairs $(X, W)$ where $X$ is a smooth compact oriented manifold of even dimension and $W$ is a complex vector bundle on $X$ :

$$
\left(X_{1}, W_{1}\right) \sim\left(X_{2}, W_{2}\right)
$$

if there exists a smooth compact oriented manifold $Y$ with boundary $\partial Y=X_{1} \cup\left(-X_{2}\right) \quad\left(-X_{2}\right.$ denotes $X_{2}$ with the opposite orientation) and a complex vector bundle $U$ on $Y$ with $U \mid X_{i} \cong W_{i}$. The set of cobordism classes forms an abelian group, the addition being given by disjoint sums. We denote this group by $A$. Then the second main step in the proof of Theorem 1 is:

Proposition 4. If $(X, W) \sim 0$ then $\mu(X, W)=0$ and $\gamma(X, W)=0$.

REMARKS. The vanishing of $\mu$ is elementary. In the case considered by Hirzebruch $(W=1)$ the vanishing of $\gamma$ follows from the topological interpretation of the index. In the general case, however, we have to give an analytical proof. The idea of the proof is as follows. Let $\partial Y=X$ and $U \mid X=W$. On $Y$ the elliptic differential operator $D=* d+d *$ operates $^{9}$ on the vector bundle $\sum_{k} \Lambda^{2 k} \otimes W$. Along the boundary $X$ this vector bundle splits into $E \otimes W$ and $F \otimes W$ of (3.1) (ii). Then

$$
\begin{array}{ll}
D u=0, & u \mid X \in F \otimes W, \\
D u=0, & u \mid X \in E \otimes W
\end{array}
$$

are well-posed boundary problems in the sense of [1] which give rise to a singular integral operator $T$ on $X$ (see [3]) such that

(i) $\gamma(T)=0$,

and

(ii) $\sigma(T)=W \sigma_{0}$.

Now, by generalizing the basic work of Thom, one can determine the group $A$ (cf. [12]). In fact for our purposes it is enough to determine $A \otimes Q$ which is somewhat easier. To complete the proof of

${ }^{\circ}$ One has to use a connection in $W$ so that $d$ will operate on forms with coefficients in $W$. 
Theorem 1, for $\operatorname{dim} X$ even, we therefore need only check that $\mu=\gamma$ on the generators of $A \otimes Q$. In fact one can prove that both $\mu$ and $\gamma$ are multiplicative, i.e., that

$$
\mu\left(X_{1}, W_{1}\right) \cdot \mu\left(X_{2}, W_{2}\right)=\mu\left(X_{1} \times X_{2}, W_{1} \otimes W_{2}\right)
$$

and similarly for $\gamma$. Using this one is finally reduced to checking the following:

(i) the Hirzebruch index of $P_{2 n}(C)$ (complex projective space) is equal to 1 ;

(ii) the Euler number of $S^{2 n}$ (the sphere) is 2.

Alternatively we observe that the generators of $A \otimes Q$ are all homogeneous bundles over homogeneous spaces so that one can use (3.4).

By using the multiplicative property of the index the case of an odd-dimensional $X$ can be reduced to that of the even-dimensional manifold $X \times S^{1}$.

6. Further remarks. (1) A more general definition of ellipticity than that used here has been given in [13]. It is not difficult to formulate this for vector bundles and show that Theorem 1 still holds.

(2) Another generalization is to introduce elliptic complexes, i.e., sequences

$$
E: 0 \rightarrow E_{n} \stackrel{D}{\rightarrow} E_{n-1} \stackrel{D}{\rightarrow} \ldots \stackrel{D}{\rightarrow} E_{0} \rightarrow 0
$$

of differential operators (of the same order) with $D^{2}=0$ and such that the sequence of symbols on $S(X)$ is exact. Examples: $D=d$ or $\bar{\partial}$. The index now gets replaced by the Euler characteristic of the cohomology of the complex $\Gamma(E)$. Theorem 1 extends to this case.

(3) In view of Theorem 3 it is natural to hope that the Grothendieck-Riemann-Roch theorem for proper maps of complex manifolds will come out of a suitable generalization of Theorem 1.

(4) The problem of extending Theorem 1 to manifolds with boundary presents some difficulties. However, using the results of [3] one can use Theorem 1 to compute the index of a large class of boundary problems.

\section{REFERENCES}

1. S. Agmon, A. Douglis and L. Nirenberg, Estimates near the boundary for solutions of elliptic PDE satisfying general boundary conditions. I, Comm. Pure Appl. Math. 12 (1959), 623-727; II (to appear). 194.

2. M. S. Agranovic, On the index of elliptic operators, Soviet Math. Dokl. 3 (1962),

3. M. S. Agranovic and A. D. Dynin, General boundary value problems for elliptic systems in higher-dimensional regions, Dokl. Akad. Nauk SSSR. 146 (1962), 511-514. 
4. M. F. Atiyah, The Grothendieck ring in geometry and topology. Proc. Internat. Congr. Math., Stockholm, 1962 (to appear).

5. M. F. Atiyah and F. Hirzebruch, Vector bundles and homogeneous spaces, Proc. Sympos. Pure Math., Vol. 3, pp. 7-38, Amer. Math. Soc., Providence, R. I., 1961.

6. - Analytic cycles on complex manifolds, Topology 1 (1962), 25-45.

7. A. Borel and F. Hirzebruch, Characteristic classes and homogeneous spaces, Amer. J. Math. 80 (1958), 458-538; 81 (1959); 315-382, 82 (1960), 491-504.

8. R. Bott, The stable homotopy of the classical groups. Ann. of Math. (2) 70 (1959), 313-337.

9. - The index theorem for homogeneous differential operators (to appear).

10. R. Brauer and H. Weyl, Spinors in n-dimensions, Amer. J. Math. 57 (1935), 425-449.

11. A. P. Calderon and A. Zygmund, Singular integral operators and differential equations, Amer. J. Math. 79 (1957), 901-921.

12. P. Conner and E. E. Floyd, Differentiable periodic maps, Ergebnisse der Mathematik, Springer, Berlin, 1963.

13. A. Douglis and L. Nirenberg, Interior estimates for elliptic systems of $P D E$, Comm. Pure Appl. Math. 8 (1955), 503-538.

14. A. S. Dynin, Singular operators of arbitrary order on a manifold, Soviet Math. Dokl. 2 (1961), 1375-1377.

15. - Multidimensional elliptic boundary value problems with a single unknown function, Soviet Math. Dokl. 2 (1961), 1431-1433. 113.

16. I. M. Gel'fand, On elliptic equations, Russian Math. Surveys 15 (1960), no. 3,

17. F. Hirzebruch, New Topologische Methoden in der algebraischen Geometrie, Ergebnisse der Mathematik, Springer, Berlin, 1956.

18. K. Kodaira, The Riemann-Roch theorem on compact analytic surfaces, Amer. J. Math. 73 (1951), 813-875.

19. R. T. Seeley, Singular integral operators on compact manifolds, Amer. J. Math. 81 (1959), 658-690.

20. - Regularization of singular integral operators on compact manifolds, Amer. J. Math. 83 (1961), 265-275.

21. - The index of elliptic systems of singular integral operators (to appear).

22. A. I. Vol'pert, On the index of systems of two-dimensional singular integral equations, Soviet Math. Dokl. 3 (1962), 154.

OXFord UNIVERSITY,

HARVARD UNIVERSITY AND

Massachusetts Institute of Technology 